\title{
Supernovae as \\ distance indicators
}

Bruno Leibundgut 


\section{Importance of SNe for $\mathrm{H}_{0}$}

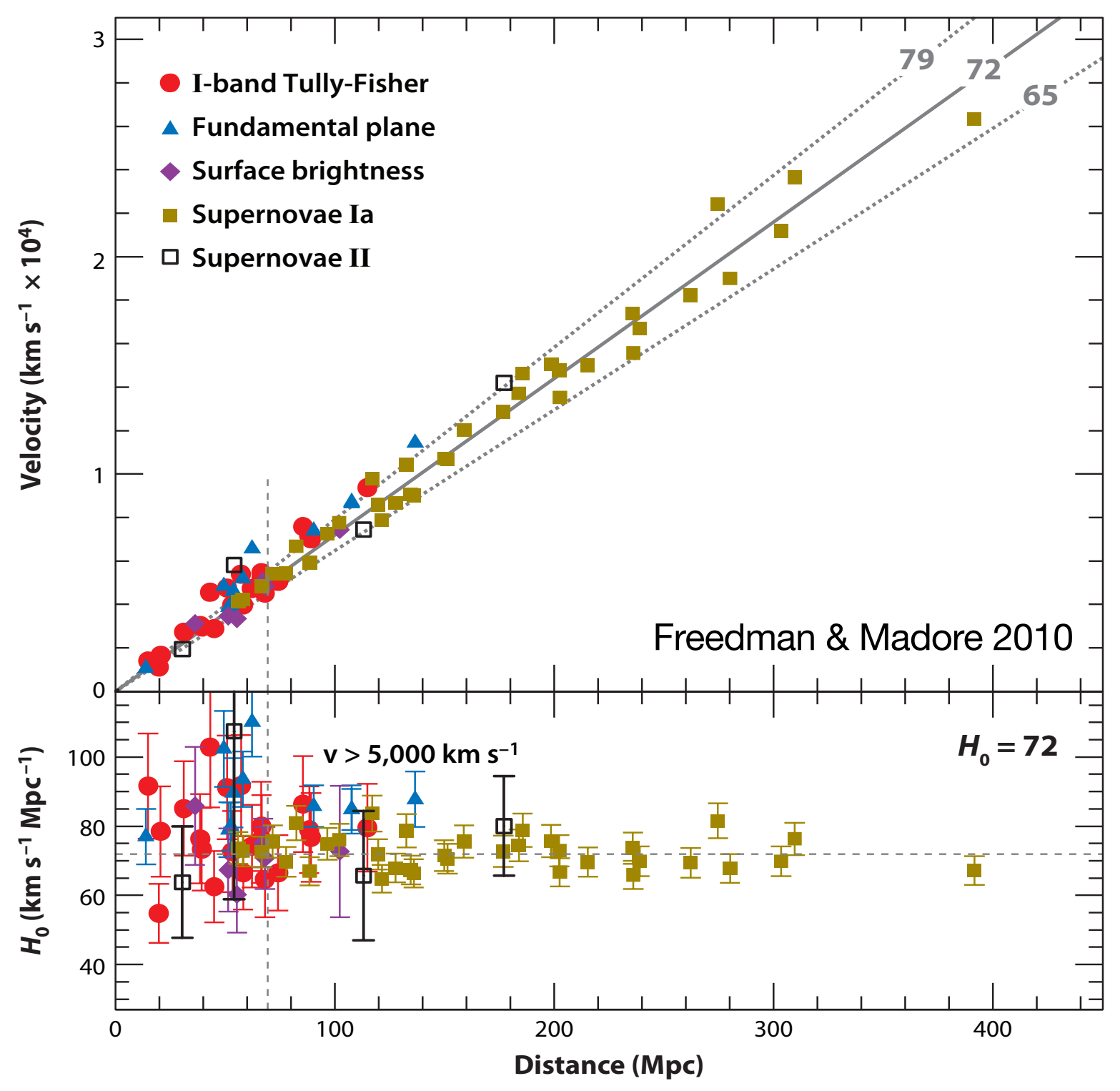




\section{Type la Supernovae}

\section{Variations on a theme}

- critical parameters?

- nickel mass

- ejecta mass

- explosion energy(?)

- explosion mechanism?

- progenitor evolution?

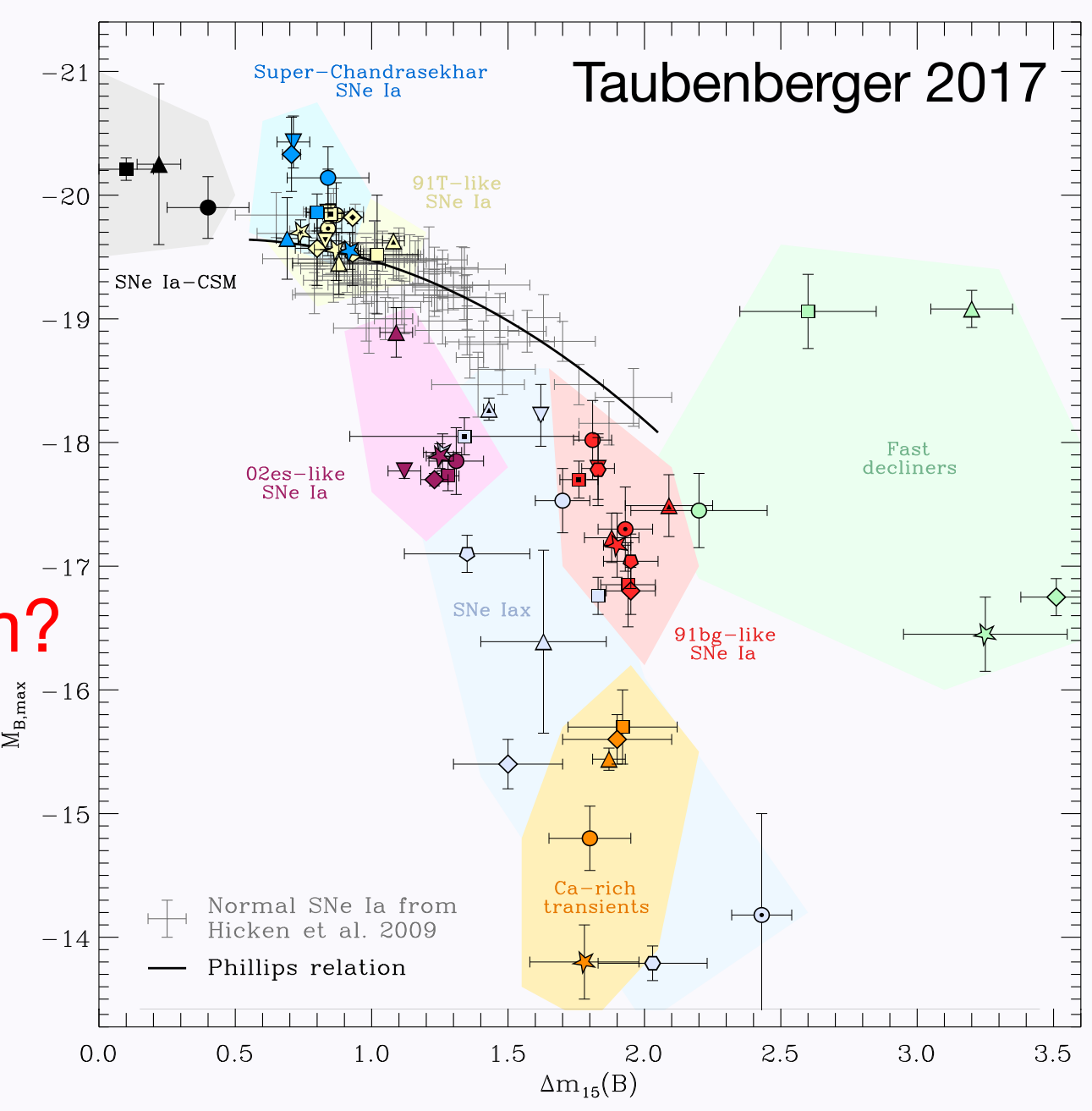




\section{Selection of SNe la for Cosmology}

Use methods to normalise the peak luminosity ("Phillips relation") - "standardization"

- SALT2, colour-stretch ( $\left.\mathrm{s}_{\mathrm{BV}}\right)$, SiFTO, etc.

- corrections for light curve shape, colour/extinction and dependence on host galaxy mass

- SALT2 (Guy et al. 2010): $\mu=m_{B}^{*}-M+\alpha X_{1}-\beta C$

- host galaxy mass dependence fit separately

- Scolnic et al. (2018), Smith et al. (2020): $\mu=m_{B}^{*}-M+\alpha x_{1}-\beta c+\Delta_{M}+\Delta_{B}$ " includes host galaxy correction and bias correction

- color-stretch (Burns et al. 2014, 2018, Freedman et al. 2019)

$$
B^{\prime}=B-P^{1}\left(s_{B V}-1\right)-P^{2}\left(s_{B V}-1\right)^{2}-R_{B} E(B-V)-\alpha_{M}\left(\log \frac{M_{*}}{M_{\odot}}-M_{0}\right)
$$

- remove outliers

- fast decline $\left(-3.0<X_{1}<3.0\right)$, colour $(-0.3<C<0.3)$, goodness of light curve fit (time of maximum and uncertainty in peak brightness)

- sometimes $3 \sigma$ clipping 


\section{Excellent Relative Distances}

Many samples now

- CSP, CfA, Foundation, SN Factory
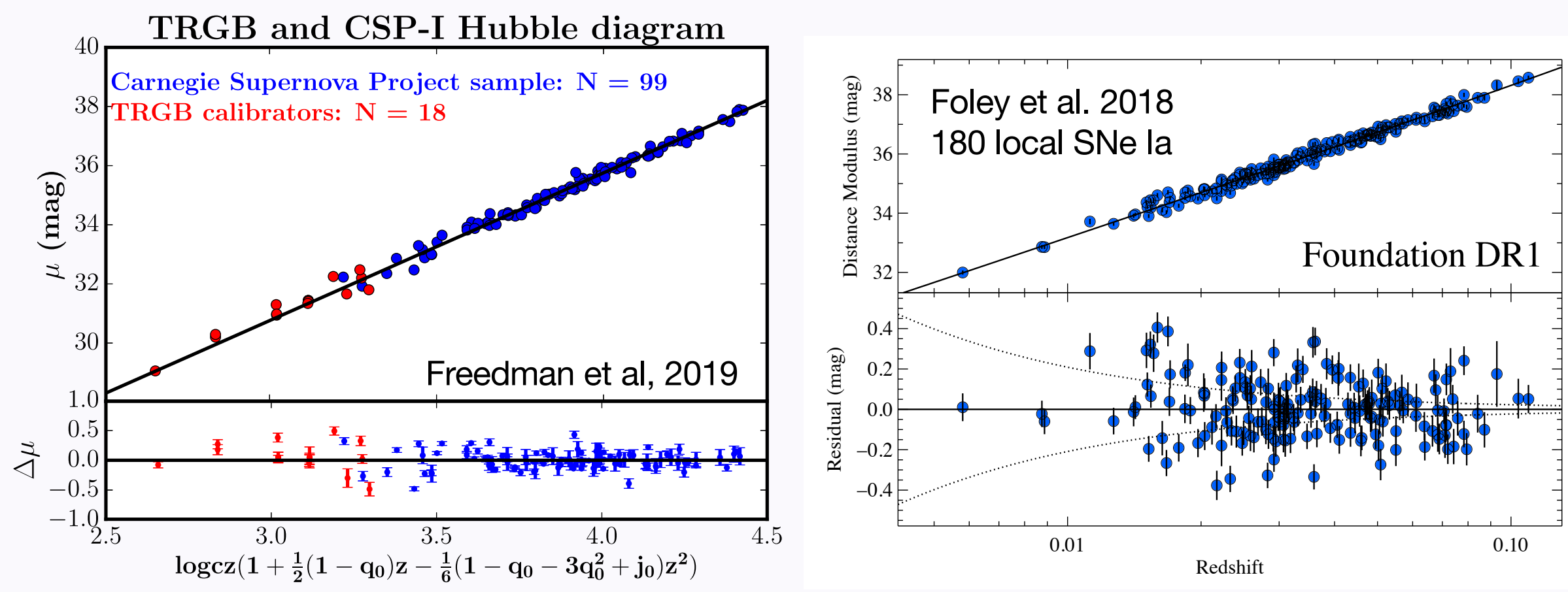


\section{$\mathrm{SNe}$ in the Near-Infrared}

"Type la are excellent standard candles in the near infrared" (Avelino et al. 2019)

Avelino et al. 2019

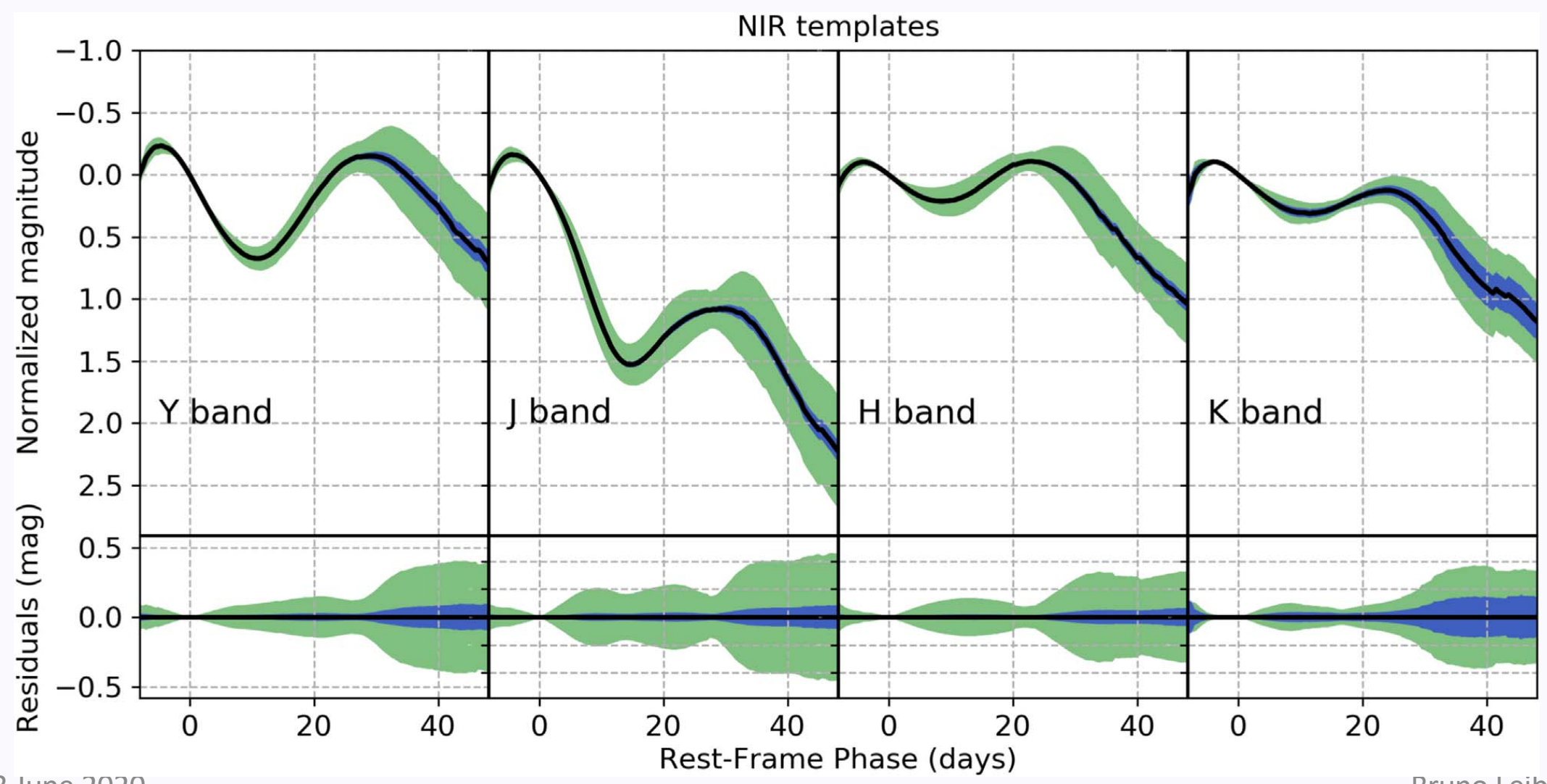




\section{SNe in the Near-Infrared}

\section{Smaller scatter in the peak magnitude}

- no corrections for light curve shape, colour or host galaxy mass

- reduced systematics due to extinction law
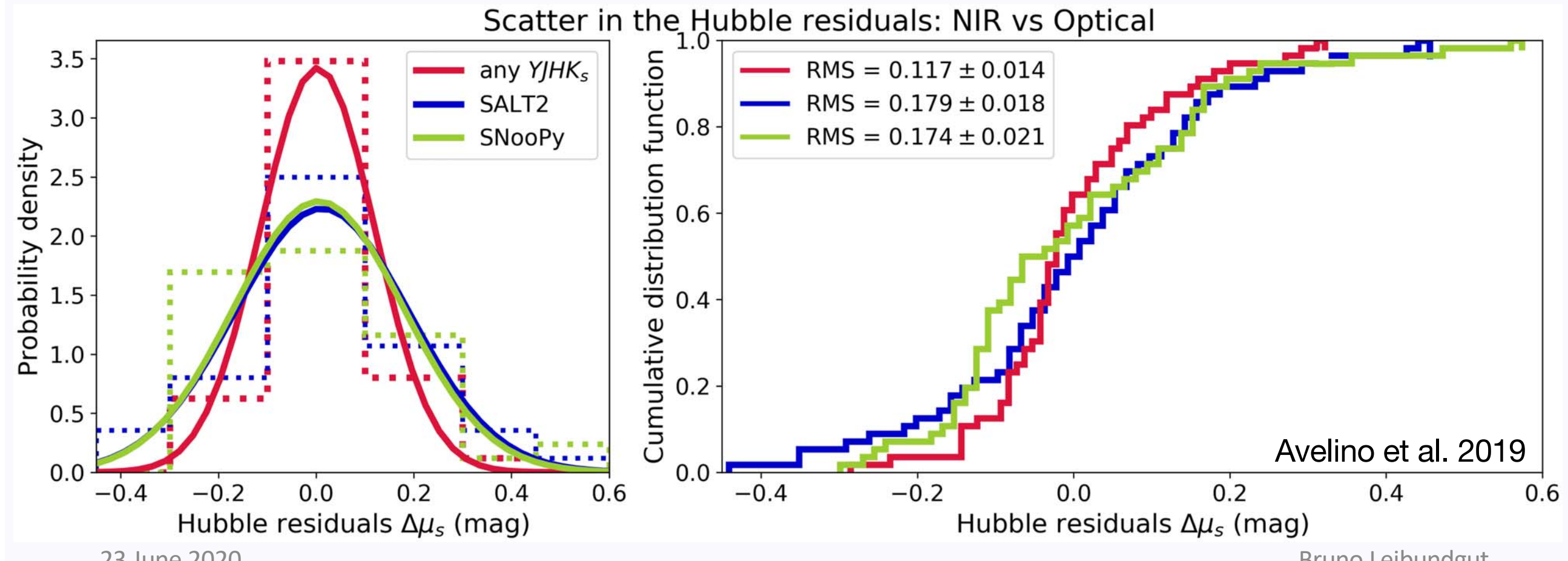


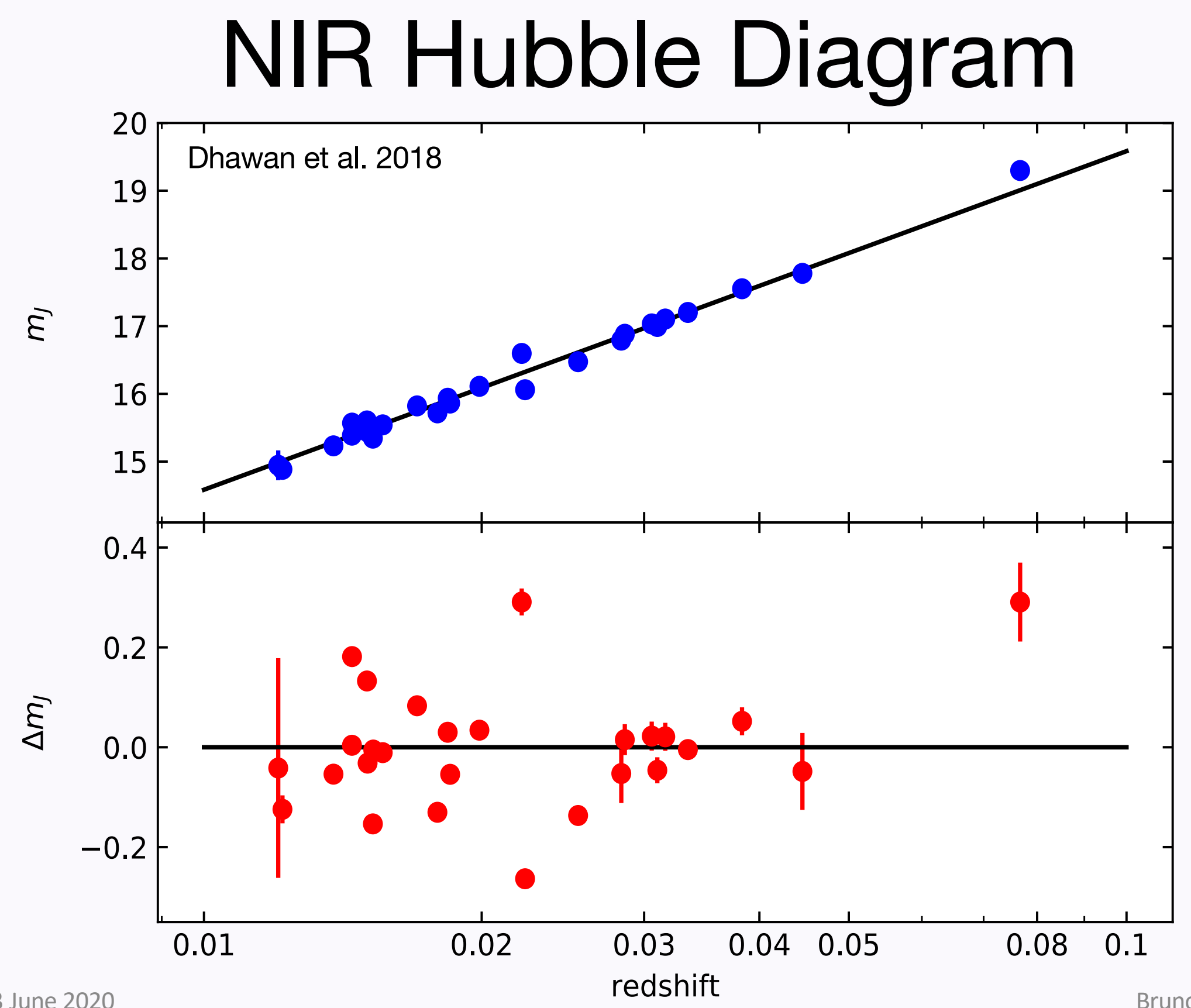




\section{NIR Hubble Diagram}

- Uncertainties with $37 \mathrm{SNe}$ la comparable to the larger ( 200 SNe la) optical data samples

- Systematic effects reduced

- fewer free parameters (light curve corrections)

- decreased influence of extinction 


\section{SNe la and $\mathrm{H}_{0}$}

- Connection into the Hubble flow

- Excellent relative distances

- 'Hubble residuals'

- Highly reliable and tested

- corrections (for optical data) under control

- $\mathrm{H}_{0}$ entirely dependent on absolute calibration of peak luminosity

- different for acceleration: based on relative distances!

- Large and reliable data samples available

- contribution to the uncertainty in $\mathrm{H}_{0}$ small

- larger NIR sample needed $\rightarrow$ CSP, RAISINS 


\section{SN la Absolute Calibration}

- Distance ladder

- Cepheids (SHOES; 74) or TRGB (70)

- BAO/Sound horizon/'inverse distance ladder'

- DES (Macaulay et al. 2019; 68)

$\rightarrow$ SNe la provide a reliable 'yardstick' and return $\mathrm{H}_{0}$ based on the calibration of their peak luminosity 


\section{Hubble-Lemaître Law}

- "Purest" way to measure: $H_{0}=\frac{C Z}{D}$

- Issues

- peculiar velocities/deviations from the Hubble flow

- problem at small distances/redshifts $(z<0.03)$

- influence of other cosmological parameters

- acceleration

- difference between linear law and $\wedge \mathrm{CDM} 4 \% @ \mathrm{z}=0.05$ 


\section{Determination of $\mathrm{H}_{0}$}

Requires knowledge of deceleration parameter

$D_{L}=\frac{c z}{H_{0}}\left\{1+\frac{1}{2}\left(1-q_{0}\right) z-\frac{1}{6}\left[1-q_{0}-3 q_{0}^{2}+j_{0} \pm \frac{c^{2}}{H_{0}^{2} R^{2}}\right] z^{2}+O\left(z^{3}\right)\right\}$

Hubble-

deceleration

Lemaître Law

- Additional uncertainty small

- less than $2 \%$ for $z<0.2$ for $0.23<\Omega_{M}<0.37$ for a flat universe

- full simulations in Dhawan et al. (2020) 


\section{Type II SNe for Cosmology}

Various methods to measure distances

- "Standardizable" Candle Method (SCM)

- Hamuy \& Pinto 2002, Olivares E. et al. 2010, de Jaeger et al. 2017, 2020

- Photospheric Magnitude Method (PMM)

- Rodríguez et al. 2019

- Photospheric Candle Method (PCM)

- de Jaeger et al. 2015 


\section{SN II SCM Hubble Diagram}

\section{Calibration through Cepheids}
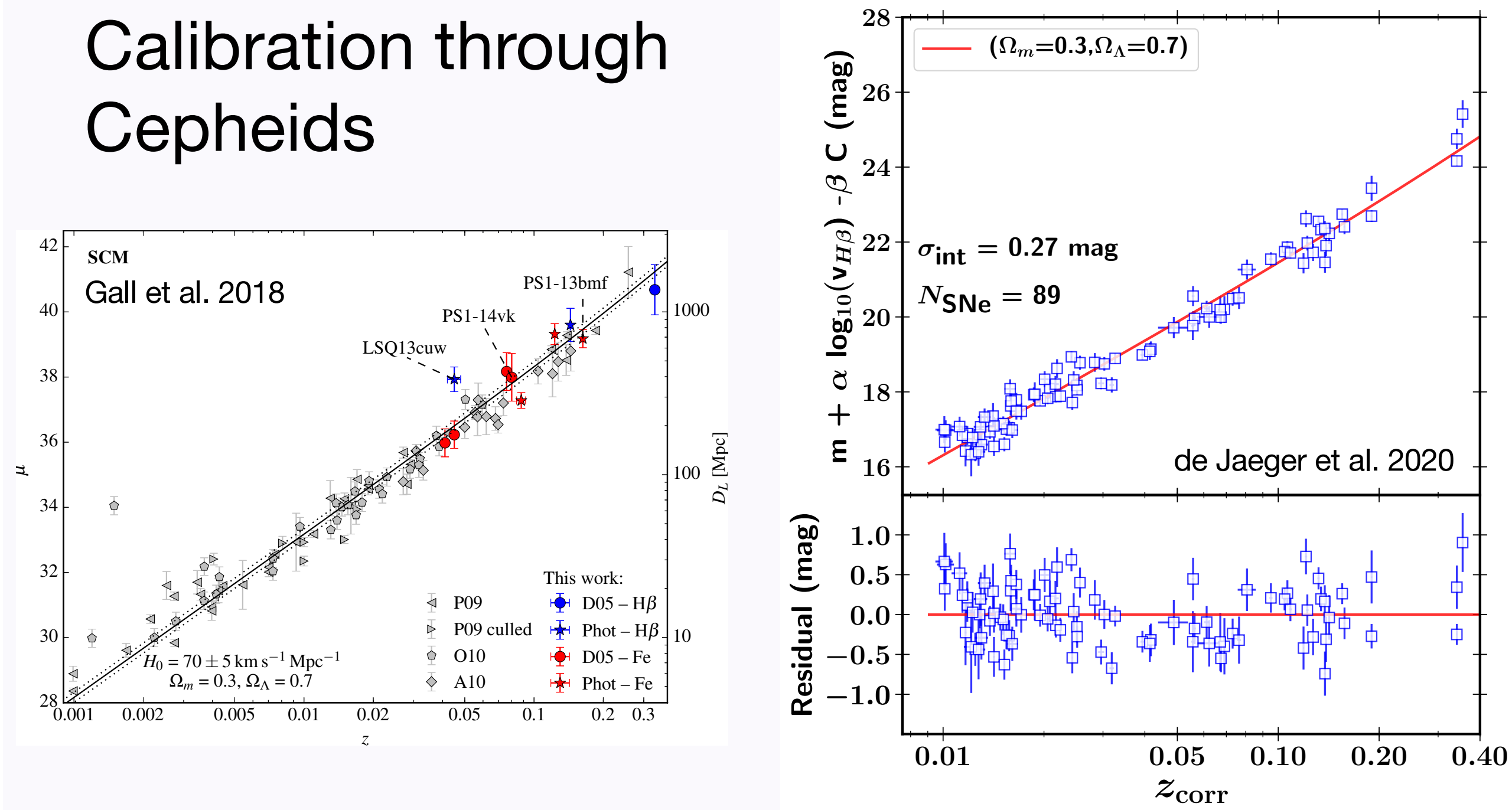


\section{Expanding Photosphere Method}

$$
\text { - } \theta=\frac{R}{D}=\sqrt{\frac{f_{\lambda}}{\zeta_{\lambda}^{2} \pi B_{\Lambda}(T)}} ; R=v\left(t-t_{0}\right)+R_{0} ; D_{A}=\frac{v}{\theta}\left(t-t_{0}\right)
$$

- $R$ from radial velocity

- Requires lines formed close to the photosphere

- $D$ from the surface brightness of the black body

- Deviation from black body due to line opacities

- Encompassed in the dilution factor $\zeta^{2}$

- Dilution factor $\zeta^{2}$ from models

- Eastman et al. (1996), Dessart (2005)

- applied to all SNe, significant differences 


\section{...not good enough}

\section{Data not constraining for accurate distances}

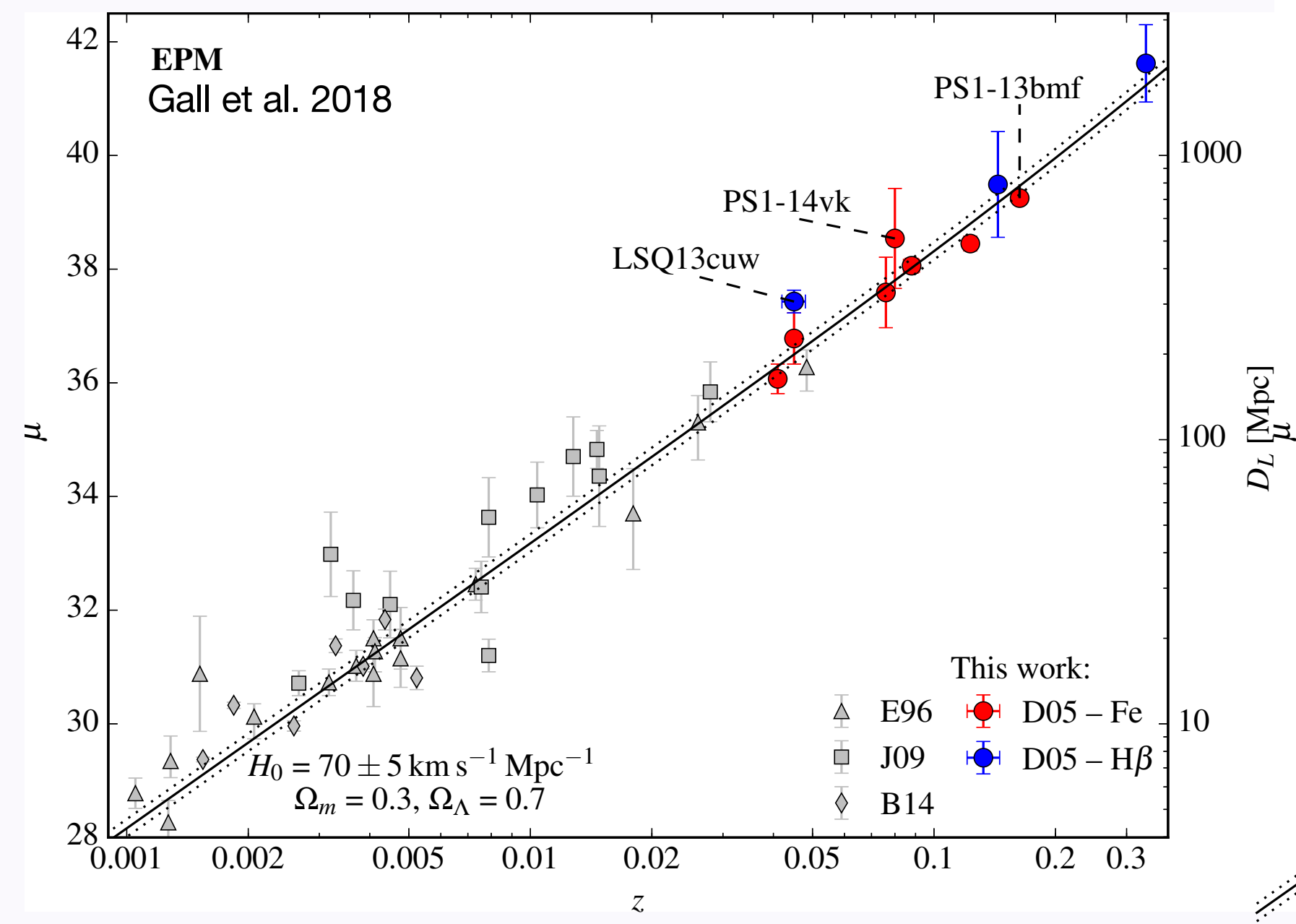




\section{Expanded Photosphere Method Reloaded}

- Use individual atmospheric models for the spectral fits

- use of the TARDIS radiation transport model

- absolute flux emitted

- Accurate explosion date

- accurate zero point

- At least 5 epochs per supernova 


\section{Atmosphere Models}
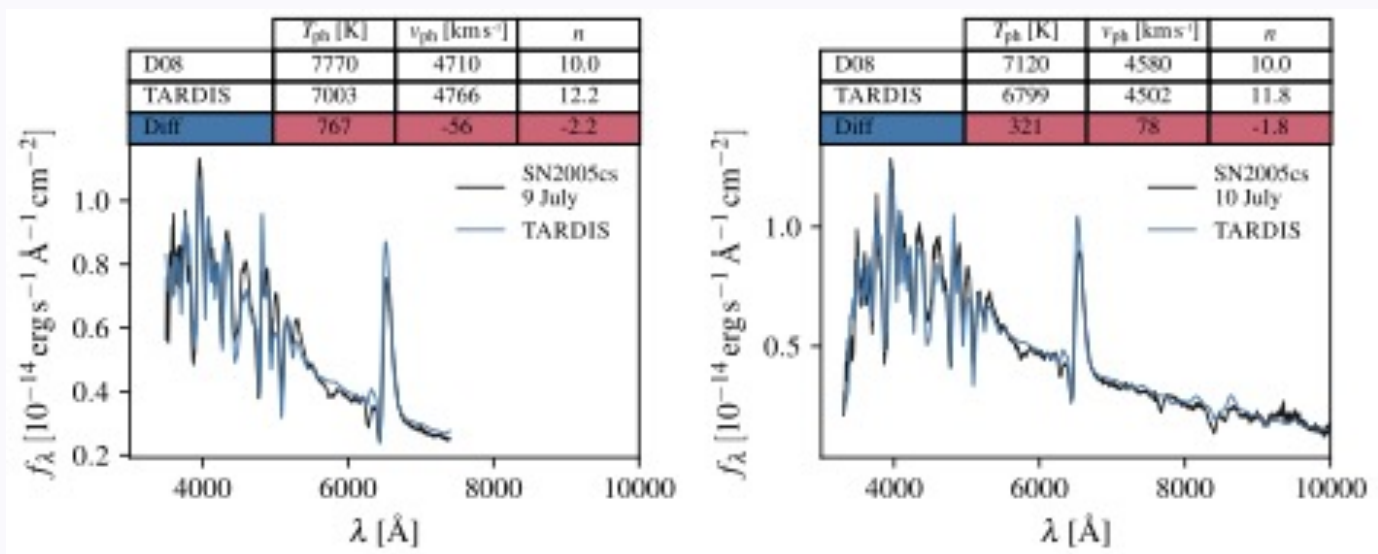

\section{TARDIS fits for different epochs}

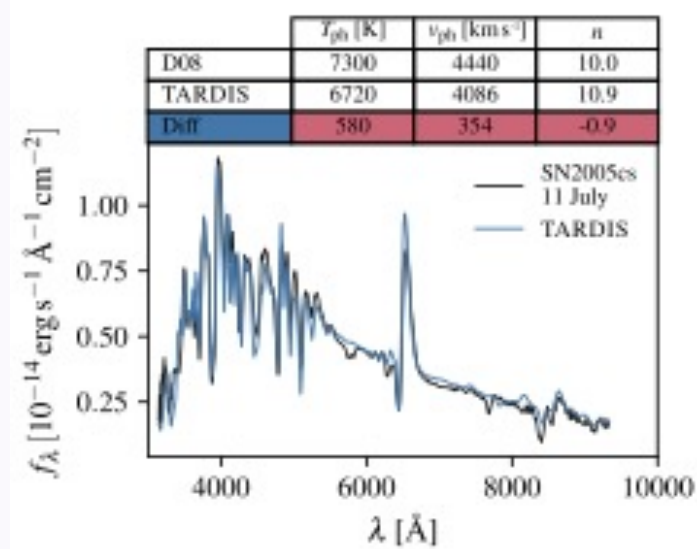

(c) 11 July 2005

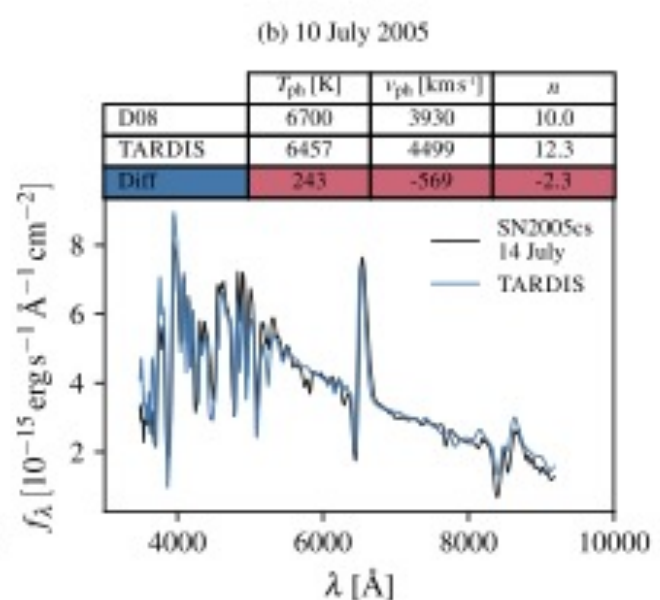

(d) 14 July 2005

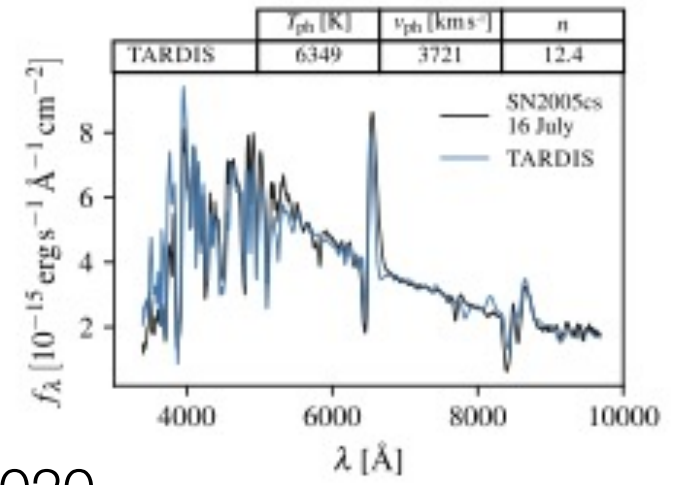

Vogl et al. 2020 


\section{Distance Determination}

Slope is inverse distance: $\frac{\Theta}{v}=\frac{1}{D_{A}}\left(t-t_{0}\right)$

$\mathrm{D}[\mathrm{Mpc}]=7.8_{-0.4}^{+0.4}$

\begin{tabular}{|c|c|c|}
\hline Date & Tume [d] & $\Theta / v_{\mathrm{ph}}[\mathrm{d} / \mathrm{Mpc}]$ \\
\hline 9 July 2005 & 12.25 & 1.69 \\
\hline 10 July 2005 & 13.50 & 1.90 \\
\hline 11 July 2005 & 14.50 & 2.12 \\
\hline 14 July 2005 & 17.00 & 2.01 \\
\hline 16 July 2005 & 19.40 & 2.44 \\
\hline
\end{tabular}

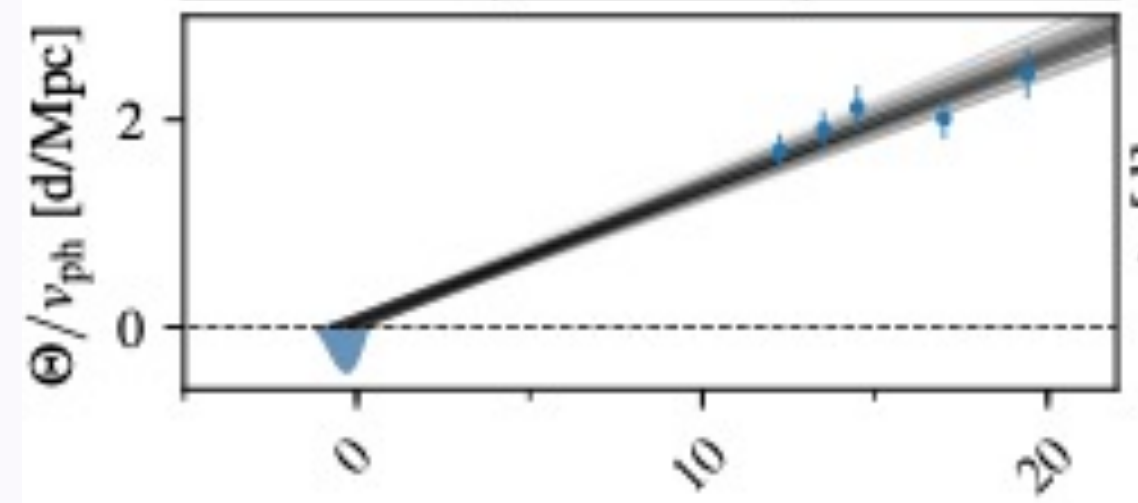

Vogl et al. 2020 Time [d]

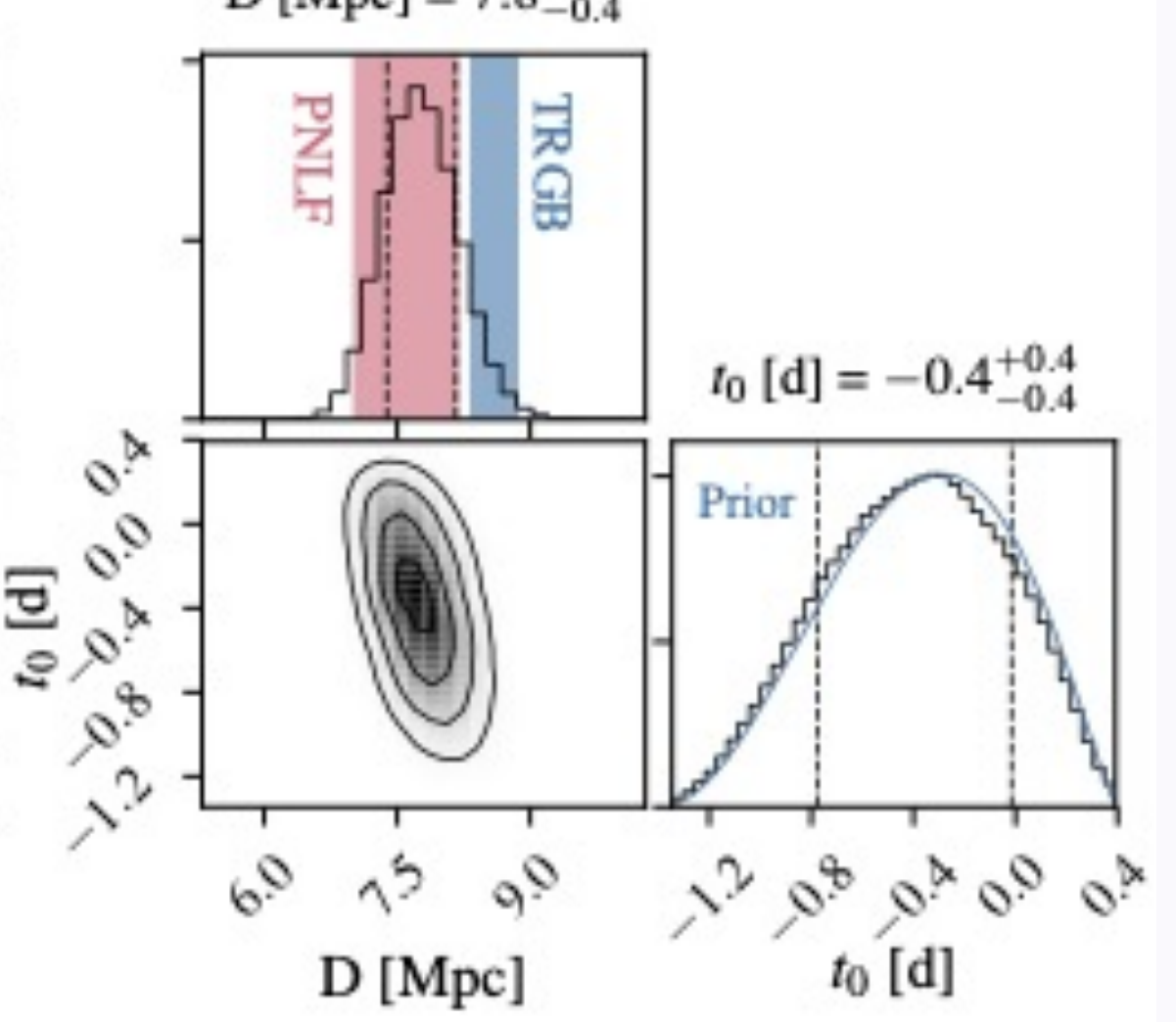




\section{Very promising...}

First evaluation of $6 \mathrm{SNe}$ from the literature

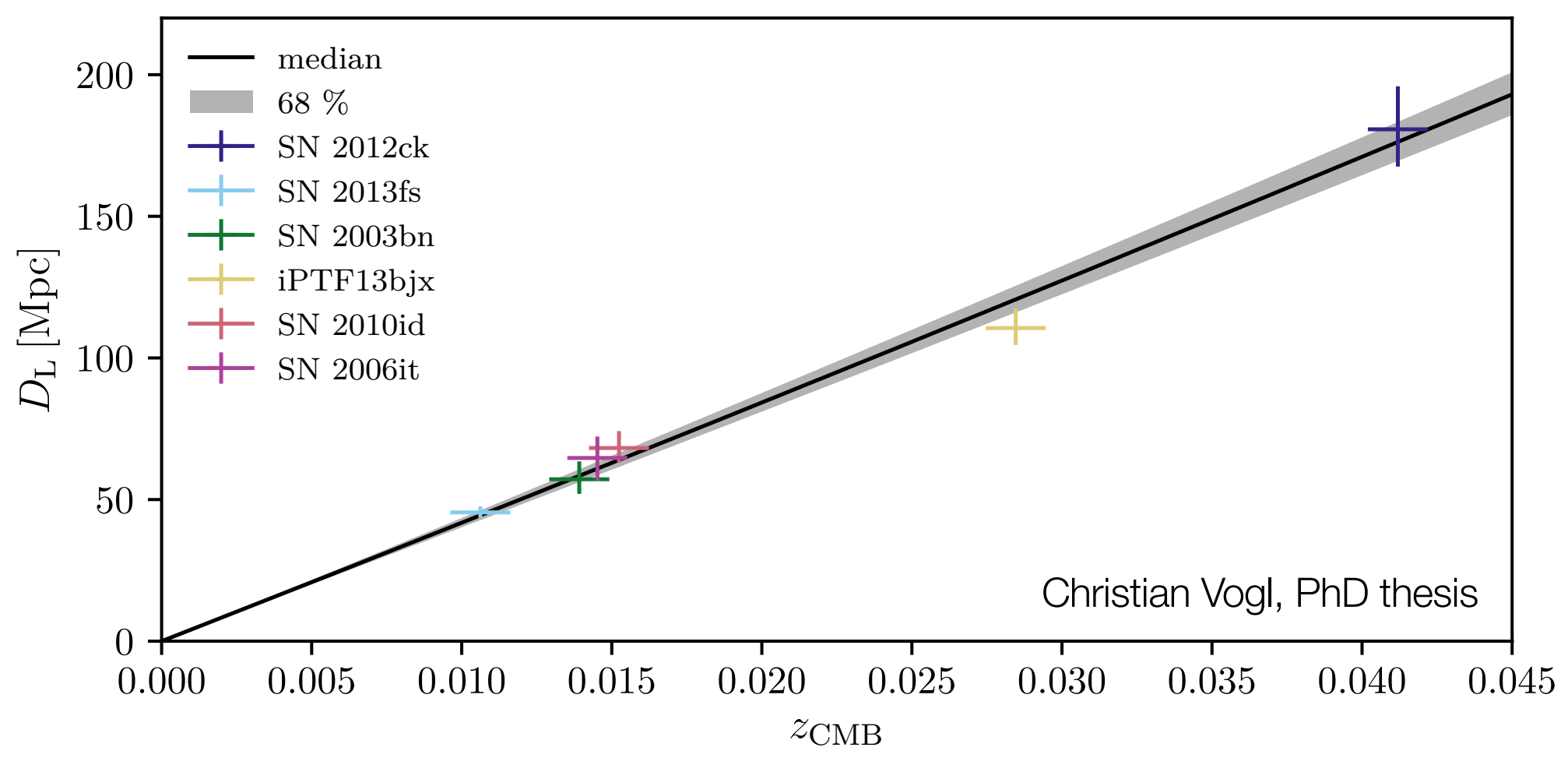




\section{adHOcc}

"accurate determination of $\mathrm{HO}$ with core-collapse supernovae"

(Flörs, Hillebrandt, Kotak, Smartt, Spyromilio, Suyu, Taubenberger, Vogl)

- Use the Expanding Photosphere Method to 30 Type II supernovae in the Hubble flow $(0.03<z<0.1)$

- Independent of distance ladder

- no parallaxes, no Cepheids, no Type la supernovae

- FORS2 Large Programme over 3 semesters

- 6 epochs spectroscopy and photometry per supernova

- $8 \mathrm{SNe}$ followed in first semester (P104)

- currently on hold

- SNFactory data

- about 15 SNe with $0.01<z<0.05$ 


\section{SN Summary}

- SNe la are reliable secondary distance indicators

- many-parameter fits/nuisance parameters

- uncertainty contributions appear controlled

- NIR promises fewer dependencies

- Combined fit for acceleration increases dependencies

- impact currently small, but might add to the $1 \%$ goal

- SNe II promising alternative

- same calibration for SCM method

- EPM completely independent

- different systematics 